\title{
Bundling effects on the intensities of second-order Raman modes in semiconducting single-walled carbon nanotubes
}

\author{
Zhengtang Luo and Fotios Papadimitrakopoulos $*, \uparrow$ \\ Nanomaterials Optoelectronics Laboratory, Department of Chemistry, Polymer Program, Institute of Materials Science, \\ University of Connecticut, Storrs, Connecticut 06269-3136, USA
}

Stephen K. Doorn*,

Chemistry Division, Los Alamos National Laboratory, Los Alamos, New Mexico 87545, USA

(Received 14 August 2007; revised manuscript received 26 October 2007; published 18 January 2008)

\begin{abstract}
The second-order Raman modes in the range of 380-650 $\mathrm{cm}^{-1}$ were investigated for individually dispersed and aggregated HiPco single-walled carbon nanotubes (SWNTs) using a 700-985 nm tunable laser source. For individually dispersed SWNTs, this Raman region displays relatively weak response from both intermediate frequency modes (IFMs) and the overtones of the radial breathing mode (RBM), with the latter dominating. In contrast, for aggregated SWNTs, the IFMs dominate and gain significant intensity relative to the RBM fundamental. Utilizing the correlation between RBM overtone and RBM fundamental intensity ratio as a function of laser energy, we derived Huang-Rhys factors for several $(n, m)$ nanotube species in both individually dispersed and aggregated states. These values were further used to obtain the corresponding absolute values of the exciton-phonon interaction matrix element for these nanotube species. It is demonstrated that the chiral-angle dependence of exciton-phonon coupling parameters is similar for dispersed and bundled samples. However, we find that bundling results in a decrease in the exciton-phonon coupling for the RBM, while the IFMs display the opposite behavior. These findings are particularly relevant for further clarifying the factors that govern Raman intensities and provide a tool for the selective characterization of various $\bmod (n-m, 3)=2$ $(n, m)$-SWNTs as a function of their aggregation state.
\end{abstract}

DOI: 10.1103/PhysRevB.77.035421

PACS number(s): 78.67.Ch, 78.66.Tr, 63.22. - m, 78.30.-j

\section{INTRODUCTION}

Single-walled carbon nanotubes (SWNTs) have attracted considerable attention from the scientific community due to their quasi-one-dimensional character and their prospective use as building blocks in electronic and optoelectronic devices. ${ }^{1}$ A number of recent reports have stressed their excitonic character as a cornerstone to their unique optical properties. ${ }^{2,3}$ The close similarity between SWNTs and conjugated organic systems allows the multiplicity of spectroscopic tools utilized for the characterization of organics to be extended to nanotubes as well. ${ }^{4}$ Resonance Raman spectroscopy has proven to be a powerful tool in probing the structural and electronic makeup of SWNTs. 5,6 In particular, the radial breathing mode (RBM, i.e., Raman shift between 100 and $380 \mathrm{~cm}^{-1}$ ) region is strongly dependent on nanotube $(n, m)$ structure, and also serves well as a sensitive probe of the physicochemical environment and aggregation state of SWNTs. ${ }^{7-12}$

Aggregation state has a profound impact on SWNT electronic behavior and observed optical properties. Theoretical descriptions of bundling interactions ${ }^{13}$ and resonance Raman measurements ${ }^{8,12}$ demonstrate significant redshifting and broadening of electronic transitions resulting from intertube interactions. Large bundle formation results in quenching of nanotube photoluminescence. ${ }^{14}$ Yet, photoluminescence is still observable within small bundles, ${ }^{15-17}$ with intertube interactions in small bundles becoming important for the emerging study of energy transfer between semiconducting nanotubes. ${ }^{15}$ Researchers have also realized the critical importance of accurate assessment of electron-phonon and exciton-phonon interactions in order to precisely predict the transport and optical behaviors of carbon nanotubes. ${ }^{2,18-20}$ As related to the nanotube Raman response, experimental ${ }^{21-23}$ and theoretical ${ }^{24-26}$ reports have revealed that the relative magnitude of the exciton-phonon interaction matrix elements $\left(M_{p h}\right)$ associated with the RBM is strongly correlated to nanotube structure, as defined by the chiral indices $(n, m)$ or its equivalent diameter $\left(d_{t}\right)$ and chiral angle $(\theta)$. With this variety of important optical and transport phenomena being driven by exciton- and electron-phonon coupling, it is of interest to gain a better understanding of how intertube interactions may impact coupling to phonon behavior in these systems.

In this work, we examine how bundling affects the electronic coupling to both the RBMs and to a particular class of intermediate frequency modes (IFMs) occurring between 380 and $420 \mathrm{~cm}^{-1} \cdot{ }^{27}$ A detailed analysis of these secondorder bands, including these IFMs and RBM-overtone Raman features, is presented for individually dispersed and bundled HiPco SWNTs in the range of 700-985 nm excitation energy. IFMs from the $2 n+m=$ const $=16$ and 19 nanotube families and RBM overtones from the $2 n+m=22$ and 25 families were found to dominate the 380-420 and $420-650 \mathrm{~cm}^{-1}$ Raman shift regions, respectively. In contrast to individually dispersed SWNTs, we find that aggregated nanotube samples exhibit a considerable increase in IFM intensity, while the RBM-overtone strength is significantly reduced. We are able to define this unique IFM behavior by, first, quantitatively evaluating the bundling effects on the exciton-phonon coupling to the RBM. While previous evaluations of RBM exciton-phonon coupling have been 
performed, ${ }^{28,29}$ here we are able to directly assess the effects of bundling on the coupling through analysis of overtone and fundamental RBM intensities in a direct comparison of individually dispersed and highly bundled samples. Resonance Raman theory derived from molecular spectroscopy indicates that the bundling-induced decrease in the RBMovertone intensity is mainly from two sources: (1) the decrease of the exciton-phonon interaction matrix element, and (2) the broadening of the optical transition width, where the latter might play a greater role. Notably, bundling is found to have a reverse effect on the IFMs. Furthermore, we also demonstrate that the chiral-angle-dependent trends observed for $M_{p h}$ of the RBM in surfactant suspended nanotubes are qualitatively preserved upon aggregation for nanotubes within the same $(2 n+m)$ family. These observations add a complementary tool to resonance Raman analysis of the RBMs for determining the complex physicochemical states of certain nanotube species.

\section{EXPERIMENTAL SECTION}

\section{A. Sample preparation}

HiPco SWNTs (batch HPR107) from Rice University were suspended in sodium dodecyl sulfate (SDS) and water, using an established method according to O'Connell et al. ${ }^{14}$ SDS (purity $>99.0 \%$ ) was purchased from Aldrich Chemicals Inc. Typically, $1 \mathrm{wt} \%$ surfactant was combined with $2 \mathrm{mg}$ of nanotube material in $10 \mathrm{ml} \mathrm{D}_{2} \mathrm{O}$, high shear mixed for $1 \mathrm{~h}$, and ultrasonicated at $\sim 500 \mathrm{~W}$ for $10-15 \mathrm{~min}$. The suspension was centrifuged for $4 \mathrm{~h}$ at $\sim 200000 \mathrm{~g}$. The resulting decant was isolated and used for subsequent experiments. Aggregated SWNT samples were prepared by adding $10 \mathrm{mg}$ of the as-produced solid HiPco SWNTs to $10 \mathrm{ml}$ of isopropanol, followed by sonication with a tip sonicator for $1 \mathrm{~min}$, followed by drop casting of the suspension on a microscope slide. An opaque SWNT film formed upon drying.

\section{B. Tunable laser resonance Raman spectroscopy}

Resonance Raman spectroscopy was performed using a tunable Ti:sapphire laser with excitation wavelengths between 695 and $985 \mathrm{~nm}$ (in 3-5 nm increments) at room temperature and ambient pressure. A SPEX triple monochromator and back-illuminated deep-depletion charge coupled device camera were used for spectral collection with $5 \mathrm{~cm}^{-1}$ resolution and $5 \mathrm{~min}$ integration times. Laser power at the sample was $20 \mathrm{~mW}$. Raw spectra were background subtracted to remove residual Rayleigh scattering and the fluorescence originating from semiconducting SWNTs (for surfactant suspended samples). Raman frequencies were calibrated at each excitation wavelength with the nonresonance Raman spectrum of 4-acetamidophenol. Intensities were corrected for instrument response using a calibration curve obtained by fitting the intensity versus excitation energy curve of 4-acetamidophenol with a third-order polynomial function.

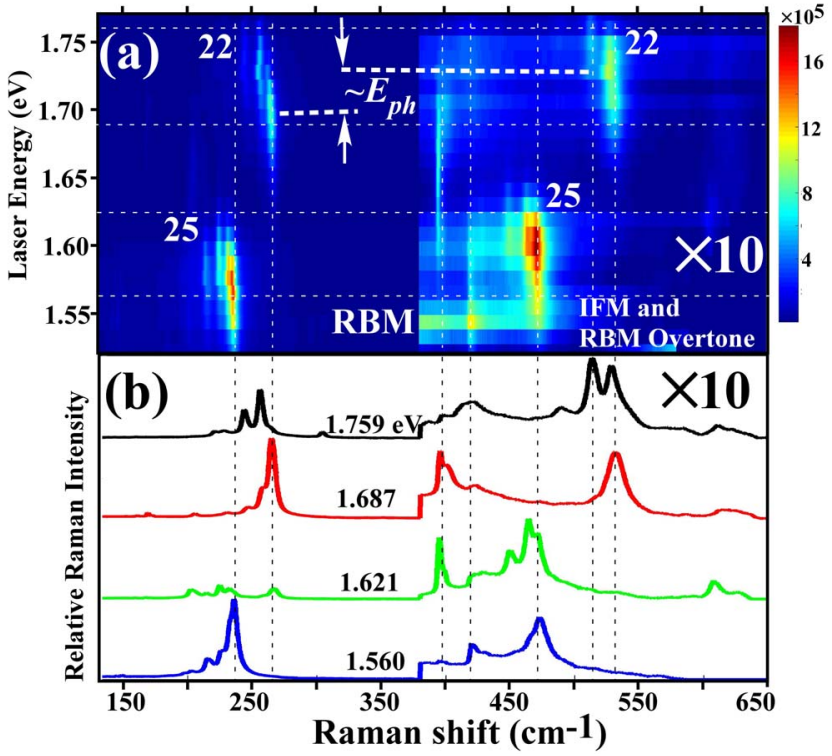

FIG. 1. (Color online) (a) Two-dimensional intensity map of laser excitation energy versus Raman shift for the RBMs, IFMs, and RBM overtones for HiPco SWNTs dispersed in SDS aqueous solution. RBM and RBM-overtone features are labeled according to corresponding $(2 n+m)$ families. (b) Representative spectra obtained with $E_{\text {laser }}=1.759,1.687,1.621$, and $1.560 \mathrm{eV}$, respectively, indicated by horizontal dashed lines in (a). Vertical dashed lines are to guide the eye with respect to corresponding features in (a) and peak positions in (b). The intensities above $380-\mathrm{cm}^{-1}$ have been multiplied by a factor of 10 in both (a) and (b) to facilitate the visualization of the weak IFM and RBM-overtone features.

\section{EXPERIMENTAL RESULTS}

Figures 1(a) and 1(b) illustrate the excitation laser $\left(E_{\text {laser }}\right.$, from 1.467 to $1.771 \mathrm{eV}$ or from 845 to $700 \mathrm{~nm}$ ) dependent Raman intensities for Raman shifts in the region of $100-650 \mathrm{~cm}^{-1}$ for individually dispersed HiPco SWNT suspensions. Resonance Raman spectra obtained with $E_{\text {laser }}$ $=1.759,1.687,1.621$, and $1.560 \mathrm{eV}$ are shown in Fig. 1(b). Spectra obtained with $E_{\text {laser }}$ in the range of $850-985 \mathrm{~nm}$ are not shown here, due to the high fluorescence background from $E_{11}$ excitonic transitions, which render the second-order Raman features nondiscernable. The RBM (Raman shift of $100-380 \mathrm{~cm}^{-1}$ ) analysis from the same $E_{\text {laser }}$ range has been published before. ${ }^{8,21,23}$ The intensities in the range of $380-650 \mathrm{~cm}^{-1}$ were multiplied by a factor of 10 to render their features comparable to the RBMs. The strong resonance behavior of RBM intensities allows us to assign 28 different semiconducting SWNTs that are in resonance with the first and second SWNT optical transitions $\left(E_{11}^{S}\right.$ and $\left.E_{22}^{S}\right) \cdot{ }^{8,21,23}$ As indicated in Fig. 1, these RBM Raman intensities are clustered into a few regions, dominated by nanotubes in the $2 n$ $+m=$ const families of 22 and 25 , as will be explained in detail later. Primarily, nanotubes of mod-2 type [defined as $\bmod (n-m, 3)=2]$ are observed, due to their stronger excitonphonon interaction matrix elements in comparison to their $\bmod (n-m, 3)=1$ (or mod-1) counterparts..$^{21,23,26,30}$ Similar data were acquired over the full excitation range on aggregated (bundled) samples. 


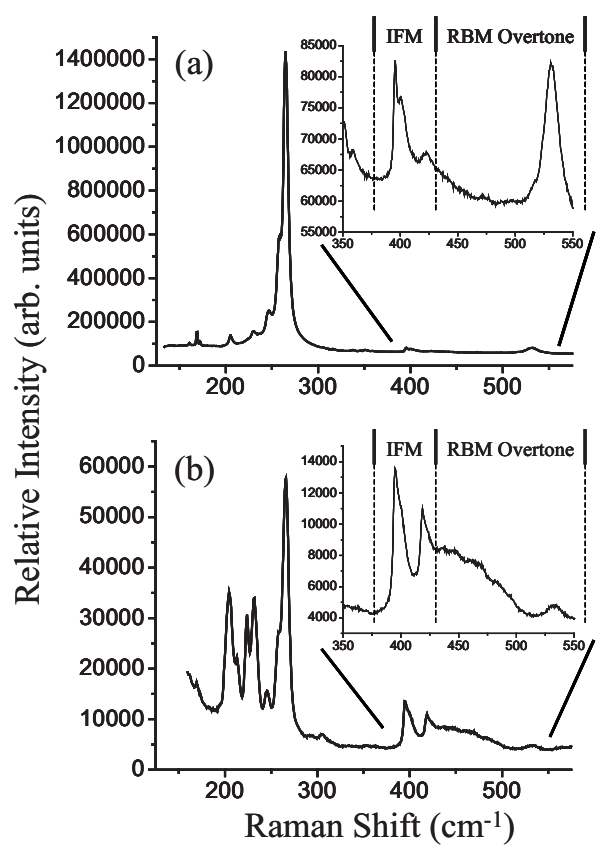

FIG. 2. Relative Raman intensities of the RBM overtone and IFM bands (inset spectra) in comparison to the RBM fundamental for (a) individually dispersed nanotube sample, $735 \mathrm{~nm}$ excitation, and (b) bundled nanotubes, $770 \mathrm{~nm}$ excitation.

In the intermediate Raman shift region $\left(380-650 \mathrm{~cm}^{-1}\right)$, we observed two types of peaks: (i) asymmetric peaks exhibiting sharp edges on the low frequency side and tails toward higher frequencies, and (ii) symmetric peaks. Two asymmetric peaks are observed with frequency maxima of 395 and $420 \mathrm{~cm}^{-1}$. In addition, these asymmetric features display a distinctive steplike dispersive behavior, characteristic of a specific class of IFMs, ${ }^{27,31,32}$ with resonances spanning from 1.73 to $1.58 \mathrm{eV}$ and from 1.63 to beyond $1.47 \mathrm{eV}$, respectively. The symmetric peaks are placed at double the Raman shift of the two RBM families (assigned to the $2 n+m=22$ and 25 families $^{8,23}$ ), indicative of their RBM-overtone nature. The RBM-overtone excitation maxima appear at higher $E_{\text {laser }}$ than that of the RBM, with an estimated difference of about $30 \mathrm{meV}$, the same order of magnitude as their phonon energies $\left(E_{p h}\right)$.

As seen in Fig. 2, significantly different bundling effects are observed for the two types of second-order modes. In Fig. 2(a), for individually dispersed SWNTs, it is seen that both the IFMs and RBM overtones are quite weak in comparison to the RBM fundamental. The overtone features are typically found to be more intense than the IFM bands in these samples. In contrast, we find that in a bundled sample, the IFMs gain significant intensity relative to the RBM fundamental [Fig. 2(b)], while the RBM overtone remains quite weak. [Note that this comparison is carried out on spectra obtained near the maxima of the $(10,2)$ resonance Raman excitation spectrum for dispersed (735 nm excitation) and bundled (770 $\mathrm{nm}$ excitation) states, respectively.]

\section{DISCUSSION}

The observed contrast in intensity behavior of the RBM overtone and IFM bands raises the following question: Is this

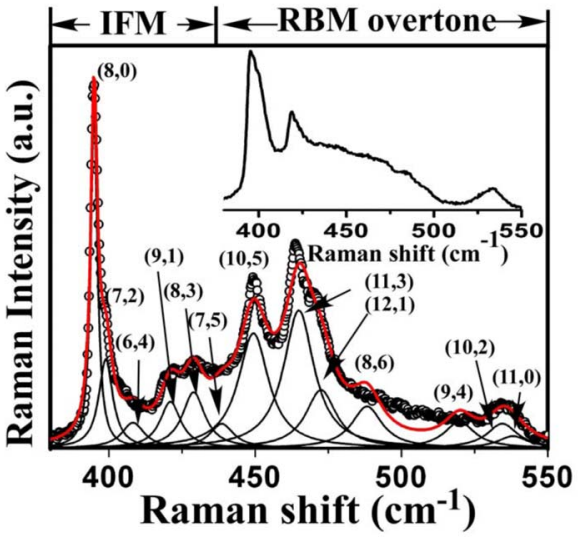

FIG. 3. (Color online) Second-order Raman spectrum obtained with $E_{\text {laser }}=1.631 \mathrm{eV}$ (or $760 \mathrm{~nm}$ ) and the deconvolution to its constituent peaks for SDS-suspended nanotubes. Experimental data are shown as open circles, with the solid line representing the combined fitted spectrum. Inset spectrum is for identical excitation of aggregated sample.

behavior due to a bundling-induced decrease in the Raman response of the RBM and its overtone, an increase in the IFM response, or a combination of the two? This question may be answered by, first, quantitatively evaluating the effect of bundling on the RBM exciton-phonon coupling and Raman intensity. The RBM may then be used as a direct intensity reference for gauging the absolute behavior of the IFM. This intensity analysis begins with deconvolution of the individual Raman spectra into specific chiral components.

For the Raman region of Fig. 1(a), each spectrum was deconvoluted using a summation of Lorentzian peak shapes, as discussed previously. ${ }^{23}$ Briefly, the combined contribution to the expected Raman intensity of all $(n, m)$-SWNTs at a specific frequency $\nu$ in the RBM spectrum can be calculated by the following equation:

$$
I\left(\nu, E_{\text {laser }}\right)=\sum_{n, m} L(\nu, \nu(n, m), \Gamma) \times A(n, m) \times \eta(n, m),
$$

where $A(n, m)$ and $\eta(n, m)$ are the abundance and Raman cross section, respectively. Function $L$ represents the Lorentzian line shape with $\Gamma$ as its full width at half maximum value. ${ }^{6}$ Figure 3 illustrates a representative Raman spectrum obtained with an $E_{\text {laser }}$ of $1.631 \mathrm{eV}(760 \mathrm{~nm})$, along with deconvolution to its constituent peaks. The major peak in the lower Raman shift region corresponds to the IFM for the $(8,0)$ zigzag SWNT, followed by progressively weaker contributions from the higher chirality $(7,2)$ and $(6,4)$ IFMs from the same family $(2 n+m=16) .{ }^{27}$ The next intensity step corresponds to the $2 n+m=19$ family, with $(9,1),(8,3)$, and $(7,5)$ nanotubes, respectively. ${ }^{27}$ Subsequently, the symmetric RBM overtones follow at higher frequencies, first from the $2 n$ $+m=25$ family [i.e., $(10,5),(11,3)$, and $(12,1)$ nanotubes] and then from the $2 n+m=22$ family [i.e., $(8,6),(9,4)$, and $(10,2)$ nanotubes]. In order to achieve convergence of the fitting for off-resonance peaks, the peak centers of less intense features are fixed at the frequency obtained in the resonance conditions. The inset of Fig. 3 depicts the Raman spectrum of an 
aggregated sample, excited at the same laser energy.

The goal in our intensity analysis is to extract the relevant exciton-phonon interaction matrix elements for both sample states in our study. These may be calculated from the HuangRhys factor $(S)$ that describes the exciton-phonon coupling within a Franck-Condon model and acts as the intensity determining factor. Equation (2) describes $S$, where $M_{p h}$ is the exciton-phonon interaction matrix element, $E_{p h}$ is the energy of the fundamental mode of the vibration (obtained from the Raman shift), and $\Delta$ is the dimensionless shift between the excited and ground states along the harmonic oscillator wave function coordinate: $:^{33,34}$

$$
S=(1 / 2)\left(M_{p h} / E_{p h}\right)^{2}=(1 / 2) \Delta^{2} .
$$

Resonance Raman spectroscopy has been employed to extract the $S$ values for a variety of organic molecules. ${ }^{35}$ More recently, such treatments have been extended to individually dispersed and weakly bundled (2-3 nanotubes) SWNTs. ${ }^{28,29}$ Huang-Rhys and $M_{p h}$ values for exciton coupling to the RBM were determined from simultaneous fitting analyses of RBM fundamental and overtone Raman excitation profiles. In principle, these methods could be applied to a determination of $S$ in fully bundled samples as well, but the method of Shreve et al. ${ }^{28}$ requires simultaneous fitting to absorption or photoluminescence excitation spectra, which are lacking for bundled nanotubes. We, therefore, introduce here an alternative approach to determining $S$, also based on a comparison of fundamental and overtone intensities for a specific phonon mode. This method can be applied selfconsistently to both dispersed and bundled excitation data in a straightforward manner. This approach is, therefore, suitable for our purpose of quantifying the differences in Raman response of the second-order modes for the dispersed and bundled systems.

In resonance Raman spectroscopy of small molecules, the Raman intensity of each fundamental vibration $\left(I_{R B M}\right.$ in our case) can be calculated by Eq. (3) based on small shift and single mode approximation assumptions: ${ }^{33,36}$

$$
I_{R B M}=\frac{C \Delta^{2} E_{p h}^{2}}{2}\left|\frac{1}{\left[E_{\text {laser }}-E_{i i}-i \gamma_{e}\right]\left[E_{\text {laser }}-E_{p h}-E_{i i}-i \gamma_{e}\right]}\right|^{2} \text {. }
$$

Here, $C$ is a normalization factor introduced to account for sampling conditions and collection geometry, and $E_{i i}$ and $\gamma_{e}$ are the energy and half-width of the optical transition, respectively.

Similarly, the small shift approximation for the first overtone of that vibration is given by Eq. (4) (Ref. 33):

$$
I_{R B M-\text { overtone }}=\frac{C \Delta^{4} E_{p h}^{4}}{2}\left|\frac{1}{\left[E_{\text {laser }}-E_{i i}-i \gamma_{e}\right]\left[E_{\text {laser }}-E_{p h}-E_{i i}-i \gamma_{e}\right]\left[E_{\text {laser }}-2 E_{p h}-E_{i i}-i \gamma_{e}\right]}\right|^{2} .
$$

By fitting the $I_{R B M}$ vs $E_{\text {laser }}$ or $I_{R B M \text {-overtone vs } E_{\text {laser }} \text {, the }}$ $E_{i i}(n, m)$ and $\gamma_{e}$ values for a particular $(n, m)$-SWNT can be obtained from Eq. (3) or (4). ${ }^{33}$ According to these equations, the RBM excitation profile should exhibit two peaks at $E_{i i}$ and $E_{i i}+E_{p h}$, while the RBM-overtone excitation profile should exhibit three peaks at $E_{i i}, E_{i i}+E_{p h}$, and $E_{i i}+2 E_{p h}$. The linewidth of these transitions, however, obscures these dualor triple-maxima profiles and leads to a single excitation maximum at $E_{i i}+1 / 2 E_{p h}$ and $E_{i i}+E_{p h}$, for the RBM and RBM overtone, respectively. Thus, the difference in peak energy between RBM and RBM-overtone excitation profiles is expected to be on the order of half the $E_{p h}$. Figure 4 illustrates the RBM and RBM-overtone excitation profiles for the $(12,1)$ and $(10,2)$ nanotubes for the (a) individually dispersed and (b) aggregated states, respectively. From the RBM spectra, the $E_{p h}$ obtained for the $(12,1)$ and $(10,2)$ nanotubes are $237 \mathrm{~cm}^{-1}(29 \mathrm{meV})$ and $265 \mathrm{~cm}^{-1}(33 \mathrm{meV})$, respectively. As can be seen from Fig. 4, regardless of the aggregation states of these chiralities, the shift between the excitation profiles for the RBMs and their overtones $(32 \mathrm{meV}$ on average) closely matches this expectation. In addition, Fig. 4 indicates that, as expected for aggregated SWNT samples, both $\mathrm{RBM}$ and RBM-overtone excitation profiles are broadened and redshifted with respect to individually dispersed SWNTs. Fitting of the excitation profiles using Eqs. (3) and (4) could be applied to fundamental/overtone pairs for six chiralities in the suspended samples and four chiralities of the aggregated sample. As seen in Fig. 4 and in Table I, the $E_{i i}$ values extracted from the data fits to these expressions show that bundling induces, on average, a redshift of $54 \mathrm{meV}$, while $\gamma_{e}$ is increased by a factor of 2 . These results are in agreement with previous reports of bundling effects on Raman excitation profiles. $8,9,12,13$

By dividing Eq. (4) with Eq. (3), one obtains a simple expression of the intensity ratio between overtone and fundamental $[\mathrm{Eq} .(5)]$ that bypasses the need to experimentally determine the constant $C$ for different aggregation states: ${ }^{33}$

$$
\frac{I_{R B M \text {-overtone }}}{I_{R B M}}=\Delta^{2} E_{p h}^{2}\left|\frac{1}{\left[E_{\text {laser }}-2 E_{p h}-E_{i i}-i \gamma_{e}\right]}\right|^{2} \text {. }
$$

More importantly, Eq. (5) affords the determination of the value of $\Delta$, which, from Eq. (2), leads to the assessment of the Huang-Rhys factor $(S)$, and henceforth, the absolute value of $M_{p h} \cdot{ }^{33}$ Here, the $I_{R B M \text {-overtone }} / I_{R B M}$ ratio needs to be corrected by a factor of $N_{p h},{ }^{29}$ where $N_{p h}=n_{p h}+1$ for Stokes Raman scattering and $n_{p h}$ is the phonon number [defined as $\left.1 /\left(e^{\left|E_{p h}\right| / k T}-1\right)\right]$, since the fundamental and its overtone intensity are proportional to $N_{p h}$ and $N_{p h}^{2}$, respectively. 

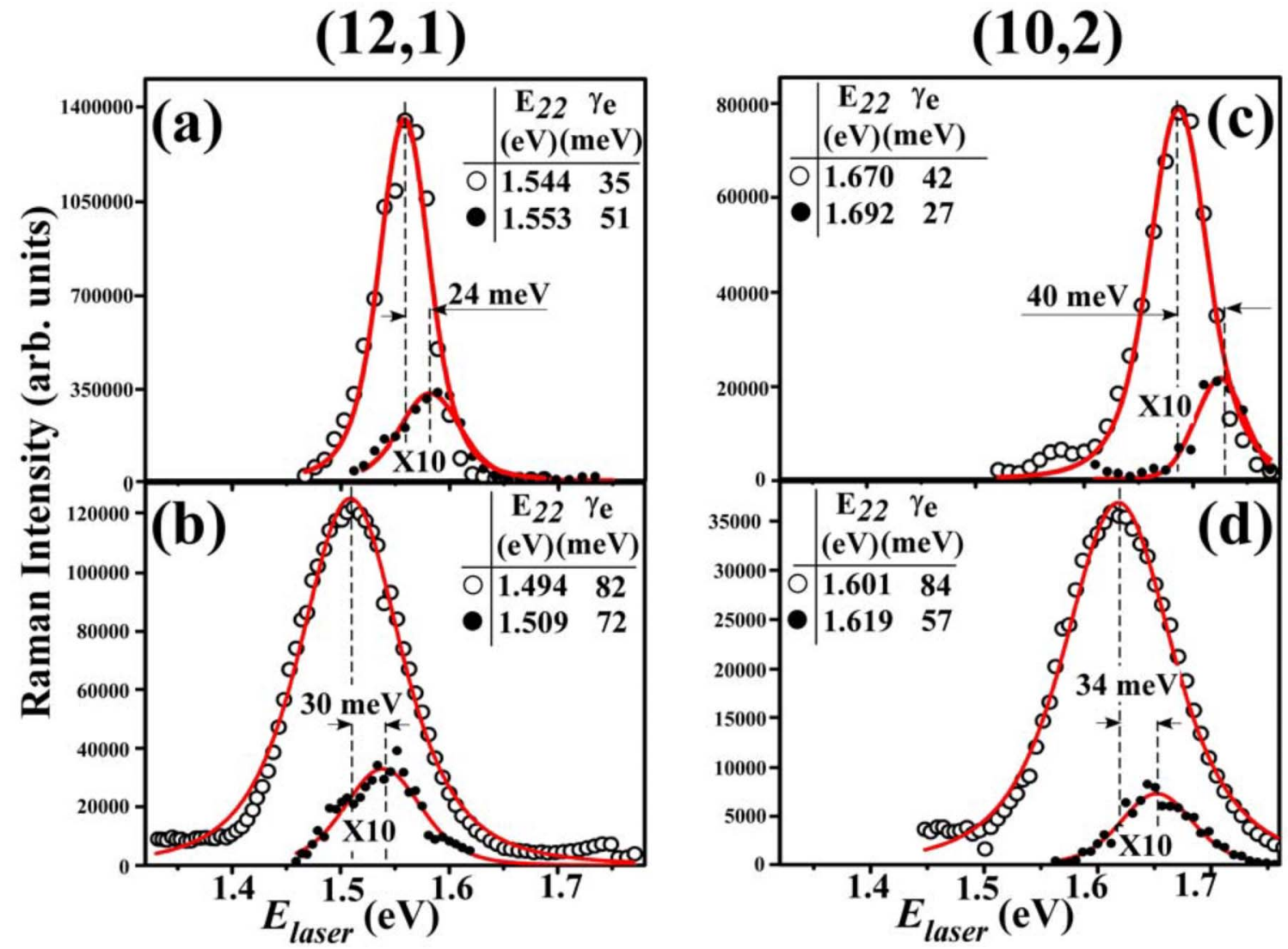

FIG. 4. (Color online) RBM (open symbols) and RBM overtone (solid symbols) excitation profiles obtained for the nanotubes (12,1) and $(10,2)$, respectively, for [(a) and (c)] individually dispersed and [(b) and (d)] bundled nanotube samples. The solid curves illustrate the fits to the RBM and RBM-overtone processes using Eqs. (3) and (4), respectively. Inset table indicates the $E_{i i}$ and $\gamma_{e}$ values utilized to obtain the corresponding fit. Note that overtone intensities have been multiplied by a factor of 10 for clarity.

Figure 5 illustrates the $I_{R B M \text {-overtone }} / I_{R B M}$ ratio as a function of laser excitation for the $(12,1)$-SWNT in (a) individually dispersed and (b) aggregated states. Equation (5) allows both $E_{i i}$ and $\gamma_{e}$ values to be independently obtained (see insets in Fig. 5). Considering the larger inaccuracies expected from the ratio analysis, we find these values to be in good agreement with the aforementioned results. When in resonance (i.e., $I_{\text {laser }}=E_{i i}+2 E_{p h}$ ), Eq. (5) can be further simplified to Eq. (6) to calculate $\Delta$ from the value of
$\left(I_{R B M \text {-overtone }} / I_{R B M}\right)_{\max }$, which is easily obtained from the fitted peak maxima in Fig. 5. While $\Delta$ can also be obtained from fitting of the $I_{R B M \text {-overtone }} / I_{R B M}$ ratio profile for each nanotube, the aforementioned peak maxima assessment enables determination of $\Delta$ when the complete RBM and/or RBM-overtone excitation profile is not available.

$$
\left(\frac{I_{R B M-\text { overtone }}}{I_{R B M}}\right)_{\max }=\frac{\Delta^{2} E_{p h}^{2}}{\gamma_{e}^{2}}
$$

TABLE I. Huang-Rhys factors $(S)$ and exciton-phonon interaction matrix elements $\left(M_{p h}\right)$ determined for various $(n, m)$ individually dispersed and aggregated nanotube samples, respectively.

\begin{tabular}{|c|c|c|c|c|c|c|c|c|c|c|c|}
\hline \multirow[b]{2}{*}{$\begin{array}{l}(n, m) \\
(n, m)\end{array}$} & \multirow[b]{2}{*}{$\begin{array}{c}d_{t} \\
(\mathrm{~nm})\end{array}$} & \multirow[b]{2}{*}{$\begin{array}{c}\theta \\
(\operatorname{deg})\end{array}$} & \multirow[b]{2}{*}{$2 n+m$} & \multicolumn{4}{|c|}{$\begin{array}{c}\text { Individually dispersed } \\
\text { SWNTs }\end{array}$} & \multicolumn{4}{|c|}{ Aggregated SWNTs } \\
\hline & & & & $\begin{array}{l}E_{22} \\
(\mathrm{eV})\end{array}$ & $\begin{array}{c}\gamma_{e} \\
(\mathrm{meV})\end{array}$ & $S$ & $\begin{array}{l}M_{e-p h} \\
(\mathrm{meV})\end{array}$ & $\begin{array}{l}E_{22} \\
(\mathrm{eV})\end{array}$ & $\begin{array}{c}\gamma_{e} \\
(\mathrm{MeV})\end{array}$ & $S$ & $\begin{array}{l}M_{e-p h} \\
(\mathrm{meV})\end{array}$ \\
\hline$(10,2)$ & 0.884 & 9.0 & 22 & 1.670 & 42 & 0.13 & 17 & 1.602 & 84 & 0.07 & 12 \\
\hline$(9,4)$ & 0.916 & 17.5 & 22 & 1.711 & 39 & 0.03 & 8 & 1.657 & 89 & 0.05 & 10 \\
\hline$(8,6)$ & 0.966 & 25.3 & 22 & 1.716 & 52 & 0.03 & 8 & 1.674 & 89 & & \\
\hline$(12,1)$ & 0.995 & 4.0 & 25 & 1.544 & 36 & 0.14 & 16 & 1.494 & 82 & 0.11 & 14 \\
\hline$(11,3)$ & 1.014 & 11.7 & 25 & 1.568 & 27 & 0.06 & 10 & & & & \\
\hline$(10,5)$ & 1.050 & 19.1 & 25 & 1.569 & 27 & 0.04 & 8 & & & & \\
\hline
\end{tabular}




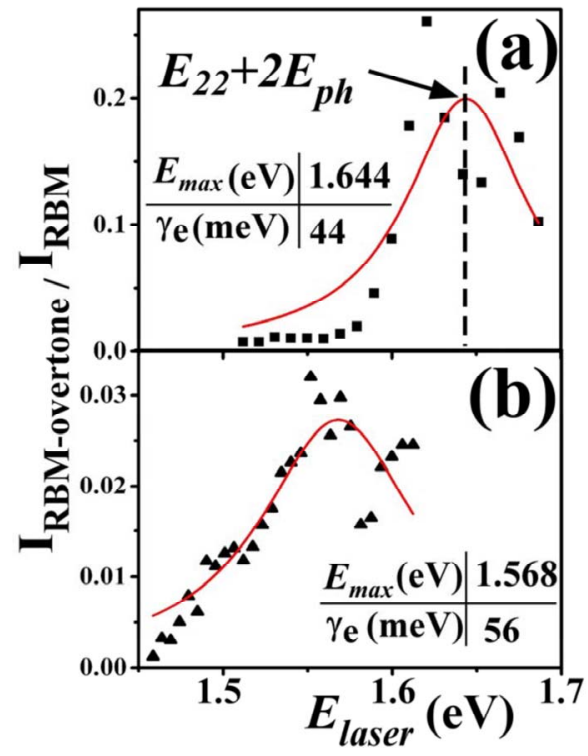

FIG. 5. (Color online) Intensity ratio of RBM overtone over $\mathrm{RBM}$ as a function of laser excitation obtained for the $(12,1)$ nanotube for (a) the individually dispersed and (b) bundled states. The obtained $E_{\max }$ and $\gamma_{e}$ values are also indicated as insets in (a) and (b).

Table I summarizes the $S$ and $M_{p h}$ values obtained for individually dispersed and aggregated SWNT samples, along with the $E_{22}$ and $\gamma_{e}$ values obtained from their RBM fundamental excitation profile fits. It is the $\gamma_{e}$ obtained from the fits to the fundamental profiles that is used to calculate $\Delta$ from Eq. (6), since, given the relative quality of the data presented, it is the fundamental profile that gives the most accurate $\gamma_{e}$ result. We estimate the error in $S$ to be on the order of $25 \%-30 \%$, with the dominant uncertainty originating in the determination of the weaker RBM-overtone intensities. The six available mod-2 nanotubes are listed with increasing diameter and chiral angle for each $2 n+m=$ const family. For all six nanotubes, their $S$ values are generally smaller than those of luminescent conjugated polymers. ${ }^{37}$ In general, the $S$ values for these polymers are in the range of 0.8-2 and are inversely proportional to the numbers of atoms in their structure, ${ }^{37,38}$ leading to the expectation of smaller $S$ values for carbon nanotubes. The small $S$ values found here are also consistent with the small Stokes shift observed for nanotube fluorescence. ${ }^{14,39,40}$

As shown in Table I, the values of the exciton-phonon interaction matrix element $\left(M_{p h}\right)$ are on the order of $10 \mathrm{meV}$ for these mod-2 nanotubes, in both the isolated and aggregated states. These $M_{p h}$ 's are comparable to those reported for individually dispersed ${ }^{28}$ and slightly bundled ${ }^{29}$ nanotubes. Closer agreement is found for the results of Ref. 29 due to similar models being used for the Raman intensity analysis. We find larger values of $M_{p h}$ in comparison with those reported in Ref. 28. The difference can be understood in that the transform method used in Ref. 28 relies on the nanotube absorbance (or photoluminescence excitation) spectrum to define the spectral line shape and to account for both homogeneous and inhomogeneous broadening mecha-

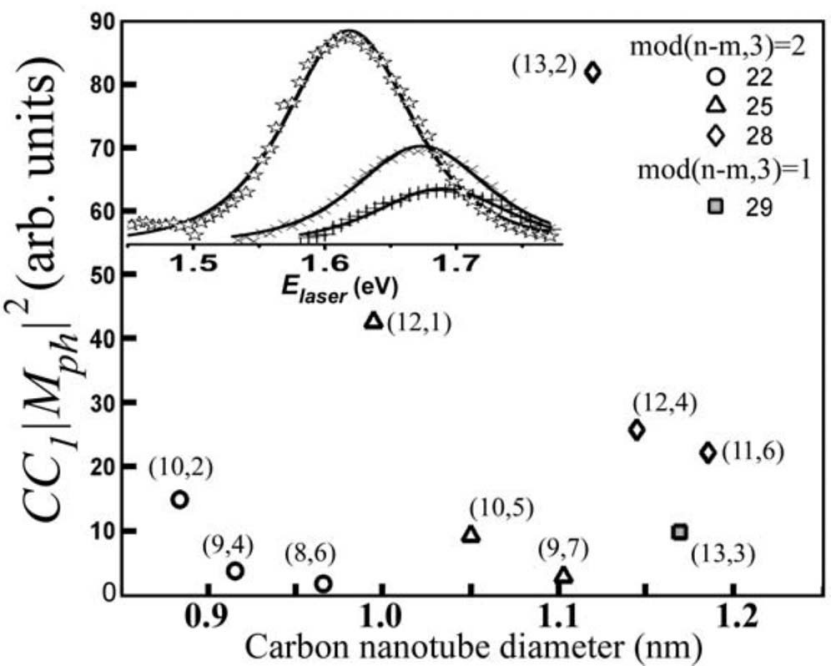

FIG. 6. Calculated $C C_{1}\left|M_{p h}\right|^{2}$ values as a function of nanotube diameter for different $(n, m)$-SWNTs in bundled sample. Open and solid symbols depict $\bmod (n-m, 3)=2$ or $1(\bmod -2$ and $\bmod -1)$ SWNT types, respectively. Nanotubes belonging to the same $(2 n$ $+m$ ) families (indicated by the number to the right of the symbol key) are plotted using the same symbol. Inset shows examples of experimental resonance windows obtained for $(10,2),(9,4)$, and $(8,6)$ (from left to right), with the solid lines showing the fitting from Eq. (3).

nisms. In contrast, the reliance of our model on the generic Raman line shape (defined by $\gamma_{e}$ ) of Eqs. (3) and (4) results in a broader fitting profile. Through Eq. (6), this translates to a larger $M_{p h}$. Additionally, non-Condon effects that were found to be important in Ref. 28 are neglected here. Such effects also ultimately impact the treatment of broadening contributions and the final profile line shape.

The $M_{p h}$ values determined from the $I_{R B M \text {-overtone }} / I_{R B M}$ analysis (Table I) for the SDS-suspended SWNTs clearly show the chiral-angle-dependent trends expected within a given $2 n+m=$ const family. In general, as the chiral angle increases within the same family of nanotubes, the $M_{p h}$ values decrease, in agreement with previous reported results. ${ }^{16,21,22,24,25,30,41}$ While we obtain fewer $M_{p h}$ values for aggregated SWNTs, a similar trend in $M_{p h}$ vs $\theta$ is observed as for the individually dispersed nanotubes. This confirms an earlier assumption that, to a first approximation, the chiralangle dependence of $M_{p h}$ is preserved upon bundling. ${ }^{23}$

To investigate in more detail the general effect of aggregation on the chiral-angle-dependent $M_{p h}$ values, we also compare the relative $M_{p h}$ values (i.e., $C C_{1}\left|M_{p h}\right|^{2}, C_{1}$ and $C$ are constant, see Ref. 23 for details) for ten SWNTs in the aggregated sample by fitting the $\eta(n, m)$ vs $E_{\text {laser }}$ curves using Eqs. (1) and (3), as widely used in many previous reports. ${ }^{21-23}$ These results are plotted in Fig. 6. The geometrical patterns for the 22,25 , and 28 nanotube $(2 n+m)$ families are clearly shown. Only one mod-1 nanotube [the $(13,3)]$ is clearly obtained, due to the weak exciton-phonon interaction expected for $E_{22}$ excitation in such groups. ${ }^{21,24,26,30}$ As with the absolute $M_{p h}$ values shown in Table I, the relative $M_{p h}$ values (Fig. 6) for the $2 n+m=22$ and 25 families follow the expected trend with chiral angle. Additionally, the relative 
values for the $2 n+m=28$ family also maintain this trend. Thus, the qualitative trend observed for these mod-2 families supports the claim that the chiral-angle-dependence of $M_{p h}$ is not affected by bundling. We note, however, that a significant difference in the diameter dependence of the relative $M_{p h}$ values appears to occur upon comparison of suspended and aggregated SWNT data. Relative values for the $2 n+m=22$ and 25 families are significantly lower than for $2 n+m=28$ in the aggregated sample. This is in contrast to the more comparable $M_{p h}$ values found for all three families in SDSsuspended samples. ${ }^{23}$ While this behavior may be due to a bundling-induced change in the diameter dependence of $M_{p h}$, it is likely that this observation instead reflects a diameterdependent preference in suspension efficiencies. Recent results have shown that the interaction between surfactant and nanotube is preferentially stabilized for smaller diameter nanotubes. ${ }^{16,42}$ Thus, relative populations of smaller diameter nanotubes may be enhanced in surfactant suspensions with respect to what is found in as-produced material.

Comparison of the $S$ and $M_{p h}$ values for suspended vs aggregated samples found in Table I shows that, in general, the $S$ and $M_{p h}$ values for the aggregated sample are lower than for the individually dispersed nanotubes, although the bundling effect is found to be weak. The decrease in the Huang-Rhys factor ranges from being on the same order as the error in the measurement to at most a factor of 2 upon aggregation. The decrease in exciton-phonon coupling may reflect either a greater exciton delocalization or restricted lattice deformation in the bundled state. Additionally, upon closer inspection of Fig. 5, one can also recognize that the $I_{R B M \text {-overtone }} / I_{R B M}$ ratios for individually dispersed SWNTs are about an order of magnitude greater than for aggregated SWNTs. This is believed to originate in part due to the $M_{p h}$ reduction (shown in Table I) and in part due to the $\gamma_{e}$ increase in the aggregated state. Since the $\gamma_{e}$ contribution is factored as the inverse square from Eq. (6), one can argue that this plays a dominant role in the decrease of the $I_{R B M \text {-overtone }} / I_{R B M}$ ratio for the aggregated state.

With the aggregation-dependent trends in exciton coupling to the RBM now determined, the RBM can serve as an intensity reference for quantifying the IFM behavior. Figure 7 illustrates IFM excitation profiles of the $(9,1)$-SWNT as a function of $E_{\text {laser }}$, along with their $E_{11}$ RBM excitation profiles, obtained for individually dispersed and aggregated nanotube samples. Due to the lack of an analytical expression for the IFM intensities vs $E_{\text {laser }}$ plot, here we simply fitted the IFM profile with a Lorentzian line shape, while for the RBM, Eq. (3) was used. As expected, the IFM excitation profiles are redshifted and broaden in width upon bundling, similar to the behavior of both excitation profiles of the RBM and RBM overtone. One can observe that the relative intensity of the IFM in the aggregated state is about 20 times larger than that for individually dispersed nanotubes. This is in stark contrast to the behavior of the RBM overtone, which, upon bundling, is reduced by about ten times. If the behavior of the $(10,2)$ nanotube is taken as an upper bound to the potential decrease in intensity of the RBM fundamental, the analysis of the previous section indicates that at most a factor of 2 of the IFM relative intensity increase is due to a

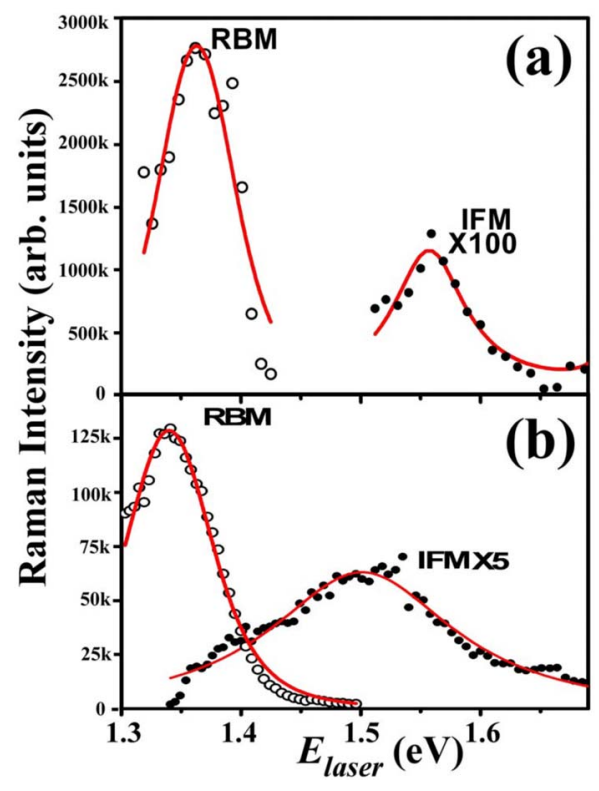

FIG. 7. (Color online) RBM (open symbols) and IFM (solid symbols) excitation profiles for $(9,1)$ in (a) the individually dispersed and (b) bundled states. The solid curves show fits with Eq. (3) and Lorentzian function for RBM and IFM excitation profiles, respectively.

loss in coupling of the RBM on bundling. Conservatively, on the order of a factor of 10 real increase in IFM intensity then remains. Thus, intertube interactions in the bundle can result in significant increases in $M_{p h}$ for the IFM. An explanation for this contrasting behavior may be found by recognizing that the involved phonons and electronic transitions for the IFM and RBM are significantly different. The IFMs studied here arise from a multiphonon scattering process ${ }^{27,31,32}$ that either resonantly connects the $E_{11}$ and $E_{22}$ transitions in a band model $^{31,32}$ or is directly resonant with formally forbidden excitonic transitions intermediate in energy between $E_{11}$ and $E_{22}$ within an excitonic picture. ${ }^{27}$ The exact nature of these modes and how they resonantly couple to the nanotube electronic structure remain an open questions.

One possible insight into their nature and why their intensity increases upon bundling may be found in recent observations that, in $\mathrm{Ar}^{+}$-ion irradiated nanotubes, IFMs also increase in intensity as the $\mathrm{Ar}^{+}$-induced defect density increases..$^{43}$ This raises the possibility that bundling acts on the IFMs in much the same way as a defect. The defects are believed to disturb the translational symmetry of the nanotube, thus relaxing the momentum conservation requirements on Raman scattering from the IFMs, which results in greater intensity. ${ }^{43}$ In much the same way, the strong intertube bundling interactions may also affect the nanotube translational symmetry to enhance the IFM intensities. Additionally, minor incorporation of metal catalyst and amorphous carbon materials (present in the raw HiPco nanotube sample) into the bundle structure might act as defect sites as well. Such a mechanism would also act to reduce RBM intensities, consistent with our observations and in agreement with the work of Ref. 43. 


\section{CONCLUSION}

In summary, we have reported experimental results for the second-order Raman intensities in the range of $380-650 \mathrm{~cm}^{-1}$, in relation to the associated RBM fundamentals, with the goal of revealing the impact of nanotube bundling on the exciton-phonon coupling in these systems. Coupling of the RBM to the excitonic transitions is found to decrease upon bundling. The possible origin of this behavior in exciton delocalization has significant implications for energy transfer in nanotube bundles. In contrast, excitonphonon coupling is found to increase for the IFMs. This behavior underscores the fundamental difference in the origin and resonance excitation behavior of the two modes. Although coupling was found to be perturbed by bundling interactions, the underlying chiral-angle dependences appear to still hold. Apparent diameter-dependent differences in ob- served relative RBM intensities on going from suspended to bundled samples parallel differences observed in suspension efficiencies for metallic versus semiconducting species in other reports ${ }^{44}$ and must be accounted for from a metrology standpoint. However, the contrasting behavior of the IFM and RBMs, especially with respect to near-zigzag chiralities, provides an additional tool for delineating the behavior of zigzag vs higher chiral-angle nanotubes for different aggregation states utilizing their second-order Raman modes.

\section{ACKNOWLEDGMENTS}

The authors would like to thank Andrew Shreve and Yan Yin for helpful discussions. Financial support from AFOSR F49620-01-1-0545 and ARODAAD-19-02-1-0381 are greatly appreciated (Z.L. and F.P.). S.K.D. acknowledges support of the LANL LDRD program.
*Authors to whom correspondence should be addressed.

†papadim@mail.ims.uconn.edu

\$skdoorn@lanl.gov

${ }^{1}$ J. Chen, V. Perebeinos, M. Freitag, J. Tsang, Q. Fu, J. Liu, and P. Avouris, Science 310, 1171 (2005).

${ }^{2}$ X. Qiu, M. Freitag, V. Perebeinos, and P. Avouris, Nano Lett. 5, 749 (2005).

${ }^{3}$ F. Wang, G. Dukovic, L. E. Brus, and T. F. Heinz, Science 308, 838 (2005).

${ }^{4}$ H. Zhao and S. Mazumdar, Phys. Rev. Lett. 93, 157402 (2004).

${ }^{5}$ A. M. Rao, E. Richter, S. Bandow, B. Chase, P. C. Eklund, K. A. Williams, S. Fang, K. R. Subbaswamy, M. Menon, A. Thess, R. E. Smalley, G. Dresselhaus, and M. S. Dresselhaus, Science 275, 187 (1997).

${ }^{6}$ M. S. Dresselhaus and P. C. Eklund, Adv. Phys. 49, 705 (2000).

${ }^{7}$ H. Kataura, Y. Maniwa, S. Masubuchi, S. Kazama, X. Zhao, Y. Ando, Y. Ohtsuka, S. Suzuki, Y. Achiba, and R. Saito, in Electronic Properties of Novel Materials-Molecular Nanostructures: XIV International Winterschool/Euroconference, edited by H. Kuzmany, S. Roth, M. Mehring, and J. Fink, AIP Conf. Proc. No. 544 (AIP, Melville, NY, 2000), p. 262.

${ }^{8}$ M. J. O'Connell, S. Sivaram, and S. K. Doorn, Phys. Rev. B 69, 235415 (2004).

${ }^{9}$ D. A. Heller, P. W. Barone, J. P. Swanson, R. M. Mayrhofer, and M. S. Strano, J. Phys. Chem. B 108, 6905 (2004).

${ }^{10}$ Z. Luo, R. Li, S. N. Kim, and F. Papadimitrakopoulos, Phys. Rev. B 70, 245429 (2004).

${ }^{11}$ Z. Luo, S. K. Doorn, R. Li, and F. Papadimitrakopoulos, Phys. Status Solidi B 243, 3155 (2006).

${ }^{12}$ C. Fantini, A. Jorio, M. Souza, M. S. Strano, M. S. Dresselhaus, and M. A. Pimenta, Phys. Rev. Lett. 93, 147406 (2004).

${ }^{13}$ S. Reich, C. Thomsen, and P. Ordejon, Phys. Rev. B 65, 155411 (2002).

${ }^{14}$ M. J. O'Connell, S. M. Bachilo, C. B. Huffman, V. C. Moore, M. S. Strano, E. H. Haroz, K. L. Rialon, P. J. Boul, W. H. Noon, C. Kittrell, J. Ma, R. H. Hauge, R. B. Weisman, and R. E. Smalley, Science 297, 593 (2002).

${ }^{15}$ O. N. Torrens, D. E. Milkie, M. Zheng, and J. M. Kikkawa, Nano
Lett. 6, 2864 (2006).

${ }^{16}$ S. Niyogi, S. Boukhalfa, S. B. Chikkannanavar, T. J. McDonald, M. J. Heben, and S. K. Doorn, J. Am. Chem. Soc. 129, 1898 (2007).

${ }^{17}$ J. Crochet, M. Clemens, and T. Hertel, J. Am. Chem. Soc. 129, 8058 (2007).

${ }^{18}$ Z. Yao, C. L. Kane, and C. Dekker, Phys. Rev. Lett. 84, 2941 (2000).

${ }^{19}$ H. Htoon, M. J. O'Connell, S. K. Doorn, and V. I. Klimov, Phys. Rev. Lett. 94, 127403 (2005).

${ }^{20}$ S. G. Chou, F. P. Filho, J. Jiang, R. Saito, D. Nezich, H. B. Ribeiro, A. Jorio, M. A. Pimenta, G. G. Samsonidze, A. P. Santos, M. Zheng, G. B. Onoa, E. D. Semke, G. Dresselhaus, and M. S. Dresselhaus, Phys. Rev. Lett. 94, 127402 (2005).

${ }^{21}$ S. K. Doorn, D. A. Heller, P. W. Barone, M. L. Usrey, and M. S. Strano, Appl. Phys. A: Mater. Sci. Process. A78, 1147 (2004).

${ }^{22}$ H. Telg, J. Maultzsch, S. Reich, F. Hennrich, and C. Thomsen, Phys. Rev. Lett. 93, 177401 (2004).

${ }^{23}$ Z. Luo, S. K. Doorn, and F. Papadimitrakopoulos, Appl. Phys. Lett. 88, 073110 (2006).

${ }^{24}$ M. Machon, S. Reich, H. Telg, J. Maultzsch, P. Ordejon, and C. Thomsen, Phys. Rev. B 71, 035416 (2005).

${ }^{25}$ V. N. Popov, L. Henrard, and P. Lambin, Phys. Rev. B 72, 035436 (2005).

${ }^{26}$ S. V. Goupalov, Phys. Rev. B 71, 153404 (2005).

${ }^{27}$ Z. Luo, F. Papadimitrakopoulos, and S. K. Doorn, Phys. Rev. B 75, 205438 (2007).

${ }^{28}$ A. P. Shreve, E. H. Haroz, S. M. Bachilo, R. B. Weisman, S. Tretiak, S. Kilina, and S. K. Doorn, Phys. Rev. Lett. 98, 037405 (2007).

${ }^{29}$ Y. Yin, A. N. Vamivakas, A. G. Walsh, S. B. Cronin, M. S. Unlu, B. B. Goldberg, and A. K. Swan, Phys. Rev. Lett. 98, 037404 (2007).

${ }^{30}$ S. V. Goupalov, B. C. Satishkumar, and S. K. Doorn, Phys. Rev. B 73, 115401 (2006).

${ }^{31}$ C. Fantini, A. Jorio, M. Souza, L. O. Ladeira, A. G. Souza Filho, R. Saito, G. G. Samsonidze, G. Dresselhaus, M. S. Dresselhaus, and M. A. Pimenta, Phys. Rev. Lett. 93, 087401 (2004). 
${ }^{32}$ C. Fantini, A. Jorio, M. Souza, R. Saito, G. G. Samsonidze, M. S. Dresselhaus, and M. A. Pimenta, Phys. Rev. B 72, 085446 (2005).

${ }^{33}$ W. L. Peticolas, L. Chinsky, P. Y. Turpin, and A. Laigle, J. Chem. Phys. 78, 656 (1983).

${ }^{34}$ S. Mukamel, Principles of Nonlinear Optical Spectroscopy (Oxford University Press, New York, 1995).

${ }^{35}$ M. Belhadj, J. M. Jean, R. A. Friesner, J. Schoonover, and W. H. Woodruff, J. Phys. Chem. 94, 2160 (1990).

${ }^{36}$ R. M. Martin and L. M. Falicov, Top. Appl. Phys. 8, 79 (1975).

${ }^{37}$ J. L. Bredas, J. Cornil, and A. J. Heeger, Adv. Mater. (Weinheim, Ger.) 8, 447 (1996).

${ }^{38}$ K. Kanemoto, T. Sudo, I. Akai, H. Hashimoto, T. Karasawa, Y.
Aso, and T. Otsubo, Phys. Rev. B 73, 235203 (2006).

${ }^{39}$ Z. Luo, L. Pfefferle, G. L. Haller, and F. Papadimitrakopoulos, J. Am. Chem. Soc. 128, 15511 (2006).

${ }^{40}$ M. Jones, C. Engtrakul, W. K. Metzger, R. J. Ellingson, A. J. Nozik, M. J. Heben, and G. Rumbles, Phys. Rev. B 71, 115426 (2005).

${ }^{41}$ S. V. Goupalov, Phys. Rev. B 72, 195403 (2005).

${ }^{42}$ T. J. McDonald, C. Engtrakul, M. Jones, G. Rumbles, and M. J. Heben, J. Phys. Chem. B 110, 25339 (2006).

${ }^{43}$ V. Skakalova, J. Maultzsch, Z. Osvath, L. P. Biro, and S. Roth, Phys. Status Solidi (RRL) 1, 138 (2007).

${ }^{44}$ C. Fantini, A. Jorio, A. P. Santos, V. S. T. Peressinotto, and M. A. Pimenta, Chem. Phys. Lett. 439, 138 (2007). 